\title{
A Thin-Plate Spline Based Multimodal Prostate Registration with Optimal Correspondences
}

\author{
Jhimli Mitra* ${ }^{*}$, Arnau Oliver $^{\dagger}$, Robert Martî ${ }^{\dagger}$, Xavier Lladó ${ }^{\dagger}$, Joan C.Vilanova ${ }^{\ddagger}$, Fabrice Meriaudeau* \\ *Laboratoire Le2I - UMR CNRS 5158, Université de Bourgogne, 12 Rue de la Fonderie, 71200 Le Creusot, France. \\ E-mail: jhimlimitra@yahoo.com, fabrice.meriaudeau@u-bourgogne.fr. \\ ${ }^{\dagger}$ Computer Vision and Robotics Group, University of Girona, Campus Montilivi, Edifici P-IV, \\ Av. Lluís Santaló, s/n, 17071 Girona, Spain. \\ E-mail: aoliver@eia.udg.edu, marly@eia.udg.edu, llado@eia.udg.edu. \\ $\ddagger$ Clinica Girona, Calle Joan Maragall 26, 17002 Girona, Spain. \\ E-mail: kvilanova@comg.es.
}

\begin{abstract}
Accurate extraction of prostate biopsy samples during Transectal Ultra Sound (TRUS) guided prostate biopsy is facilitated with the registration of pre-acquired Magnetic Resonance (MR) images with the Ultrasound (US) images. This paper proposes a novel method of generating optimal correspondences to register the MR and US images using Thin-Plate Splines (TPS) transformation. The correspondence generation method exploits the prostate shape geometry in both the modalities and is fully automatic. Normalized Mutual Information (NMI) is employed for the quantitative determination of optimal number of correspondences in terms of maximization of registration similarity. Qualitative registration results, that conform to the NMI measures are also shown for different numbers of correspondences. Shepard's interpolation method is used with the TPS in order to deal with the interpolation error of backward TPS transformation. The accuracy of our method of correspondence generation is qualitatively evaluated in comparison with two intuitive geometric contour sampling methods. An average Dice Similarity Coefficient (DSC) value of $0.97 \pm 0.01$ for 4 patient datasets is obtained for the TPS registration using our novel method of correspondences.
\end{abstract}

Index Terms-Prostate biopsy, multimodal registration, automatic correspondences, thin-plate splines, normalized mutual information.

\section{INTRODUCTION}

Prostate cancer among men has been of serious concern in Europe for the last twenty years. A patient with increased level of Prostate Specific Antigen (PSA) in blood is advised for a prostate biopsy that aids in the diagnosis of benign hypertrophy or cancer. The biopsy procedure is often guided by TRUS imaging when, clinicians generally extract 10-12 samples strategically from different regions of the prostate gland. However, the risk of the malignant prostate tissues remaining uncovered increases with this approach since the US images do not provide enough contrast between healthy and malignant prostate tissues [1]. Hence, pre-acquired MR prostate images of the same patient with better qualities and contrasts can be registered with the interventional US images to guide the biopsy procedure [2]-[5].

The prostate may undergo deformations due to the insertion of the endorectal probe through the rectum during the MR imaging, inflation of the endorectal balloon, bowel and gas in rectum, full bladder or different patient positions on the couch during the imaging procedures. Non-rigid or deformable registration deals with such prostate deformations between the modalities. However, deformable registration employing TPS transform, requires selection of correspondence points on the moving and reference images for the computation of the transformation parameters. Some researchers like Lu et al. [6] and Fei et al. [7] have used manually selected correspondences to warp MR prostate volumes with TPS registration.

In this paper, we propose a novel approach to establish an optimal set of correspondence points automatically in the US and MR modalities to achieve deformable multimodal prostate registration employing TPS. Salient points on prostate contour were automatically identified using equal angle sampling by Padilla-Castañeda and Arámbula-Cosío [8]. Yan et al. [9] used equally spaced salient points on prostate contour based on Euclidean distance to build a partial active shape model for segmentation. Our new approach of generating correspondence points exploits the principal shape components of the segmented prostate contours. The correspondences are generated in different levels termed as resolutions in the remaining paper. The optimal resolution of correspondences is determined by the maximum NMI value after the TPS registrations of all resolutions. The TPS framework includes the correspondence localization errors and uses Shepard's interpolation [10] to yield a smooth transformation. The accuracy of our correspondence point generation algorithm for registration is evaluated against two intuitive geometric approaches of prostate contour sampling. 4 patient datasets are used to validate the results. The registration results with our approach of generating correspondences shows improved performance over the other geometric approaches.

The remaining paper is organized as follows. Section II describes the two geometric sampling methods and the novel correspondence method implemented. Section III compares the results of different correspondence point generation algorithms in terms of registration quality and demonstrates the role of the NMI values in determining the optimal set of correspondences generated with our new method along with the improved registration quality using Shepard's interpolation over backward 


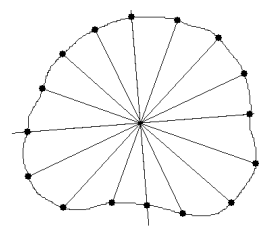

(a) Equal-angle sampling

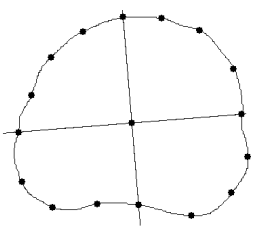

(b) Equal-space sampling
Fig. 1. Geometric methods for correspondence points generation.

TPS interpolation. Conclusions and future works are presented in section IV.

\section{PROPOSED METHOD}

This section is divided into three subsections. The first one dealing with three correspondence point generation method, the second one explaining the use of NMI in determining the optimal set of correspondences and the last one explaining the thin-plate splines with Shepard's interpolation.

\section{A. Automatic point correspondences}

The 2D MR slice corresponding to the US slice is manually resliced from the MR volume and the prostates in both the images are automatically segmented using the method of Ghose et al. [11]. The US image is treated as the reference and the MR as the moving image. Principal Component Analysis (PCA) of the segmented contour in the reference US image provides the principal axes of the prostate. These axes are then projected on the center of gravity of the prostate MR image. The intersection points of the principal axes with the prostate boundaries for both the reference and moving images are identified automatically. All correspondence point generation methods described hereafter, are based on the principal axes of the prostate.

The algorithms for geometric prostate contour sampling at equal angles and at equal spaces are implemented. In equalangle sampling method, the angles are equally spread inside each quadrant formed by the principal axes. The equallyspaced contour sampling points are also generated inside each quadrant depending on the number of contour points falling in the same. Fig. 1(a) and Fig. 1(b) show the contour points obtained with equal-angle sampling and equal-space sampling approaches respectively.

Our approach of correspondence point generation is based on triangulated approximation of the prostate quadrants. The triangulation method begins by traversing the intersections of the principal axes in a clockwise or anti-clockwise manner in each image. Let $p_{i} \mathrm{~s}, i=1, \ldots, n, n=4$ for resolution $l=0$, represent the the four intersections of the principal axes with the prostate contour. With the final resolution $L$, the algorithm is as follows

1) Resolution $l=1$.

2) Loop while $l<=L$.

3) Generate midpoint $q_{i}$ between $p_{i}$ and $p_{i+1}$ as $\left(p_{i}+\right.$ $\left.p_{i+1}\right) / 2$.

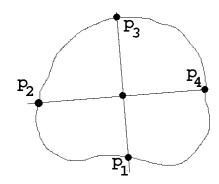

(a) Level 0

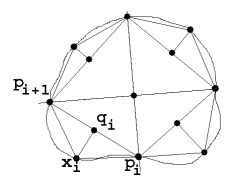

(b) Level 1

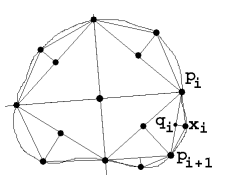

(c) Level 2, only shown for right-

bottom quadrant
Fig. 2. Method of generating correspondence points in different resolutions.

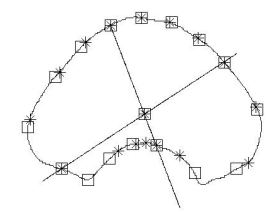

(a) Points generated in US

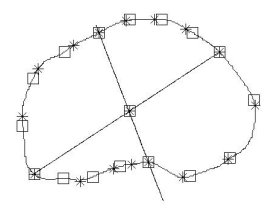

(b) Points generated in MR
Fig. 3. The $*_{s}$ indicate points generated with equal-angle sampling and the squares indicate the points generated with our approach. Note that in the bottom-left quadrant, our method is able to get good correspondences even in the presence of a significant deformation.

4) Find a point $x_{i}$ on the contour between $p_{i}$ and $p_{i+1}$ such that $\operatorname{slope}\left(p_{i}, p_{i+1}\right)$.slope $\left(x_{i}, q_{i}\right)=-1$.

5) $\left(p_{i}, x_{i}, p_{i+1}\right)$ comprise the triangulated region of the prostate between $p_{i}$ and $p_{i+1}$.

6) Repeat Steps 3-5 until $p_{i}=p_{n}$ and $p_{i+1}=p_{1}$.

7) If $l<=L$, then update $n=2 n$ and $l=l+1$, save $p_{1}, x_{1}, p_{2}, \ldots, p_{n-1}, x_{n-1}, p_{n}, x_{n}$ as new $p_{i}$ s with $i=$ $1, \ldots, n$ and repeat from Step 3. Else, end the loop.

The order of traversing the $p_{i}$ s should be the same for both the reference and moving images. Fig. 2 shows the triangulation method for three subsequent levels/resolutions.

Instead of considering only the contour points for the deformable registration, certain points inside the prostate contour are also considered for a smooth deformation of the internal glandular structures of the prostate that are quite evident in MR images and sometimes partially visible in US images. The internal points are primarily the $q_{i} \mathrm{~s}$ generated in resolution $l=1$ and the prostate centroid (see Fig. 2(b)).

As a comparison of our correspondence method to other geometric methods, Fig. 3 shows a case when bad correspondences are generated with equal-angle sampling due to significant deformation between the reference and moving images.

\section{B. NMI in determining optimality of correspondences}

NMI is an information theoretic method of measuring mutual dependence between two random variables and is commonly used as a similarity measure to achieve multimodal image registration [12].

$$
N M I(R, M)=\sum_{r, m} p_{R M}(r, m) \log \frac{p_{R}(r)}{p_{M}(m)}
$$

where, $p_{R M}(r, m)$ is the joint pdf of the reference and the moving images. $p_{R}(r)$ and $p_{M}(m)$ are the marginal pdfs of 
the reference and the moving images respectively. In this work, correspondence points are generated in different resolutions using our algorithm described in section II-A. TPS registration is then performed at each resolution of correspondences and the NMI between the deformed and reference images is computed. The resolution corresponding to the the maximum NMI is then considered as the one with the optimal set of correspondences.

\section{Thin-plate splines registration}

The thin-plate spline is a commonly used basis function in 2D-Euclidean space [13] to map the coordinates of a moving image into a reference image, when a set of homologous correspondence points are established in both images. In its extended form, the deformable TPS model includes the affine model as a special case. If $p_{i}=\left(x_{i}, y_{i}\right)$ and $q_{i}=\left(x_{j}, y_{j}\right)$, $i=j=1, \ldots, n$ represent two sets of corresponding landmarks in the moving and reference images respectively, then, the TPS interpolation $f(x, y)$ minimizes the bending energy

$$
I_{f}=\iint_{\Re^{2}}\left(f_{x x}^{2}+2 f_{x y}^{2}+f_{y y}^{2}\right) d x d y
$$

and has the form

$$
\begin{aligned}
& f(x, y)=a_{1}+a_{x} x+a_{y} y+ \\
& \sum_{i=1}^{n} w_{i} U\left(\left\|\left(x_{i}, y_{i}\right)-(x, y)\right\|\right),
\end{aligned}
$$

where, $U(r)=r^{2} \log r, a_{1}, a_{x}$ and $a_{y}$ are the affine parameters and $w_{i}$ s are the TPS parameters and $f(x, y)$ should have square integrable derivatives if

$$
\sum_{i=1}^{n} w_{i}=0 \quad \text { and } \quad \sum_{i=1}^{n} w_{i} x_{i}=\sum_{i=1}^{n} w_{i} y_{i}=0 .
$$

The boundary conditions and introduction of the correspondence localization errors in terms of regularization [14], yields a linear system of equation for the TPS coefficients as

$$
\left[\begin{array}{cc}
K+n \lambda C^{-1} & P \\
P^{T} & O
\end{array}\right]\left[\begin{array}{l}
w \\
a
\end{array}\right]=\left[\begin{array}{l}
q \\
o
\end{array}\right]
$$

where, $K$ is a $n \times n$ matrix and $K_{i j}=$ $U\left(\left\|\left(x_{i}, y_{i}\right)-\left(x_{j}, y_{j}\right)\right\|\right)$, $i$ th row of $P$ is $\left(1, x_{i}, y_{i}\right), O$ is a $3 \times 3$ matrix of zeros, $o$ is a $3 \times 1$ column vector of zeros, $w$ and $q$ are column vectors of $w_{i}$ s and $q_{j}$ s respectively, $a$ is a column vector of the affine parameters $a_{1}, a_{x}$ and $a_{y}$ and

$$
C^{-1}=\left(\begin{array}{ccc}
\sigma_{1}^{2} & & 0 \\
& \ddots & \\
0 & & \sigma_{n}^{2}
\end{array}\right) \text {. }
$$

The covariance $\sigma_{i}^{2}$ is the sum of the covariances of the points $p_{i}$ and $q_{i}$ and $\lambda=0.01$ is the regularization term. Introducing the term $n \lambda C^{-1}$ yields a better conditioned linear system and a robust numerical solution. Finally, (4) is framed as

$$
L U=V
$$

and solved as

$$
U=L^{-1} V
$$

where,

$$
L=\left[\begin{array}{cc}
K+n \lambda C^{-1} & P \\
P^{T} & O
\end{array}\right], U=\left[\begin{array}{l}
w \\
a
\end{array}\right]
$$

and

$$
V=\left[\begin{array}{l}
q \\
o
\end{array}\right]
$$

The TPS and affine parameters obtained from the TPS framework are used to warp the moving image using nearestneighbor interpolation to map the pixel values onto integer coordinates. However, TPS interpolation produces holes in the transformed image as all its pixels are not being mapped. Therefore, a general approach is to unwarp the transformed image to fill up the unmapped pixels. Unfortunately, the backward transformation fails to achieve accurate results since, splines are not exactly reversible. Therefore, we used Shepard's interpolation [10] to deal with these unmapped pixels. This method of interpolation successfully interpolates a point from scattered pixel points. In our method, a $7 \times 7$ mask is centered on an unmapped pixel and the intensity of the unmapped pixel is interpolated from the mapped pixels within the mask. Such interpolation produces smooth intensity warping of the moving image and is faster than computing the reverse TPS parameters.

\section{RESUlts AND Discussions}

The US images used in our experiments are acquired by Siemens AQUSON and the MR slices are obtained from a GE 1.5 Tesla machine. 4 patients middle slice images of the prostate are used from which the prostates are segmented automatically. For all the MR/US image pairs, we applied the three correspondence generation methods described in section II-A. Fig. 4 shows the obtained registration results with Patient-1. Observe that our new method of correspondence points (see last row of Fig. 4) produce a better registration result than the other two approaches. In our method, the correspondences are generated from resolution 1 through 3 including the center. At each resolution a TPS/Shepard's interpolation is done and the NMI is computed. The resolution corresponding to the maximum NMI is considered as the optimal resolution. Table I shows the NMI for different resolutions for all patients. The columns pertaining to the bold figures are the optimal correspondence point resolutions for the respective patients. Fig. 5 shows the different registration results with different resolution of correspondences for Patient-4. It must be noted that the results are in conformity with the NMI values in the last row of Table I. The maximum NMI and the best registration quality for Patient-4 are obtained for the 3rd resolution of correspondences as evident from both the table and the figure.

Fig. 4 and Fig. 6 show only the optimal set of correspondences using our approach. Notice from the columns 1 and 2 of 

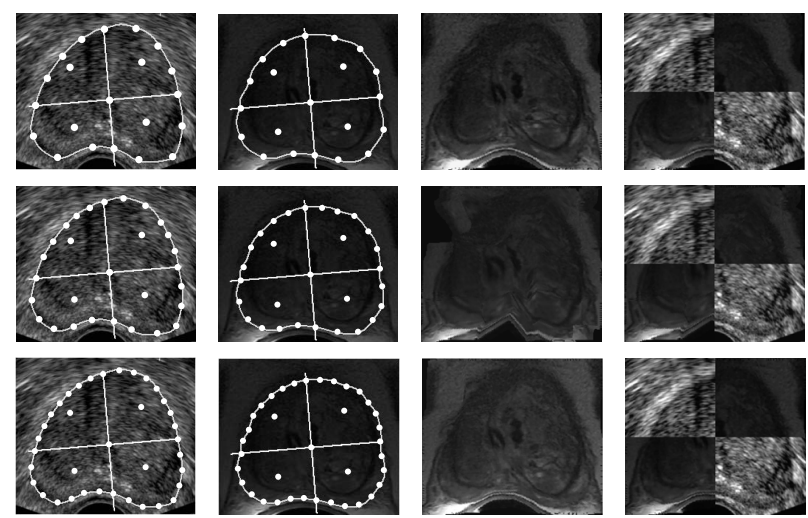

Fig. 4. Qualitative registration results for Patient-1. (First-row) correspondences using equal-angle sampling, (second-row) correspondences using equal-space sampling, (third-row) correspondences using our approach. The first-left column shows the reference US images, second column comprises of the moving MR images, third column shows the registered images and the last column shows checker-board displays to evaluate the quality of registration.
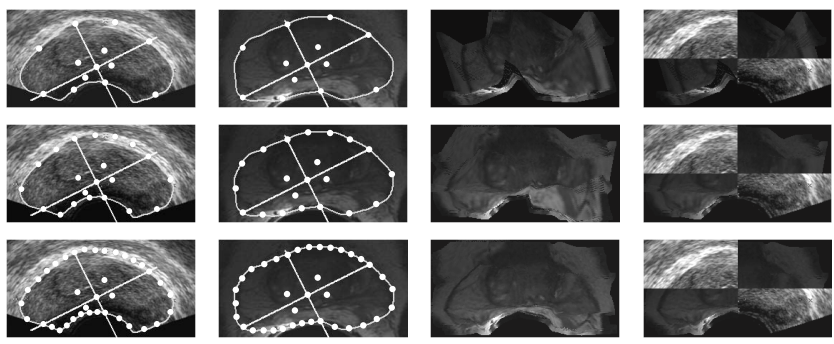

Fig. 5. Patient-4 quality of registrations for different resolutions of correspondences using our new method. (First-row) Level 1 correspondences, (second-row) level 2 correspondences, (third-row) level 3 correspondences. The column representations are the same as Fig. 4.

Fig. 4 that the number of points differ for each correspondence generation method. With our method a maximum of 37 correspondences (level 3) yields a smooth deformation. On the other hand with other geometric correspondence methods, less number of correspondences provide satisfactory results and an increase in the number of points increase the correspondence localization errors resulting in undesirable registrations. This is well justified with the fact that our method estimates correspondence points in localized regions and thus, can avoid undesirable localization errors.

It is to be observed from Fig. 4 (1st and 2nd rows), that the overall accuracies of registration along the prostate boundaries are satisfactory when equal-angle and equal-space samplings are used respectively, although, the internal structures do not have smooth deformations with equal-angle sampling. Equalangle sampling produces large correspondence localization errors for Patient- 2 and Patient- 4 and hence, TPS registrations are unsatisfactory. Therefore, the NMI approach to determine optimal correspondences is not applied for equal-angle and equal-space contour sampling methods. Fig. 6 shows the results obtained with our new correspondence generation method for patients 2, 3 and 4 respectively.

Dice Similarity Coefficient (DSC) is used to evaluate the
TABLE I

NMI AT DIFFERENT RESOLUTIONS.

\begin{tabular}{|c|c|c|c|}
\hline \hline Patient\# & Level 1 & Level 2 & Level 3 \\
\hline 1 & -260.24 & -174.23 & $\mathbf{- 1 6 7 . 6 7}$ \\
2 & -781.48 & $\mathbf{- 4 1 2 . 0 1}$ & -424.14 \\
3 & -419.79 & $\mathbf{- 4 0 8 . 5 4}$ & -408.68 \\
4 & -454.60 & -403.03 & $\mathbf{- 3 8 9 . 4 5}$ \\
\hline
\end{tabular}
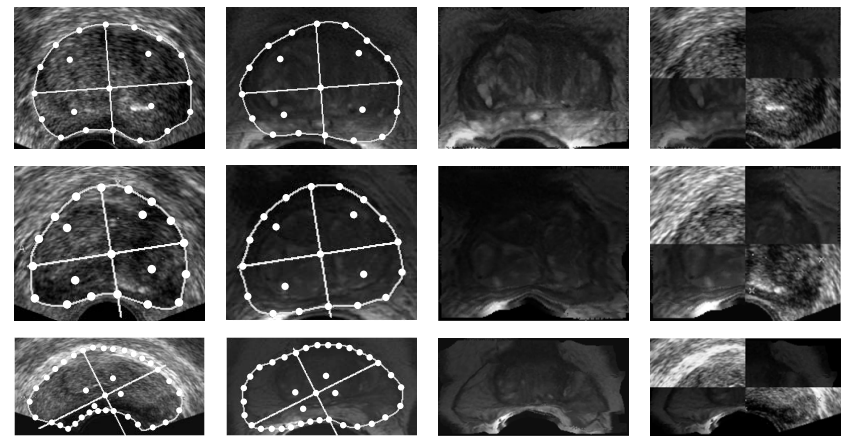

Fig. 6. Patient-2 to Patient-4 registration results using our correspondence generation method. The columns are similar to Fig. 4.

registration accuracy that measures the overlap between the transformed image and the reference image. The choice of DSC over other registration error measures is due to the fact that most registration errors involve marking targets manually on the reference US and the moving MR images. Even if the targets are easily visible on the MR image, the corresponding targets may not be visible in the US image due to low contrast. Also, manual selection of targets introduces intra- and interobserver localization errors and may not provide accurate registration error. Table II shows DSC values obtained with our method along with the computation times for generating correspondence points and TPS interpolation with Shepard's interpolation. An average DSC of $0.97 \pm 0.01$ is obtained for all the patients. The equal-angle, equal-space sampling and our method perform satisfactorily on patients 1 and 3 and the DSC values of only Patient-1 after TPS registration are $0.97,0.96$ and 0.98 respectively. Therefore, it may be inferred that despite the high DSC values provided by the geometric sampling methods in certain cases, our method consistently performs better for all patient cases.

Fig. 7 shows the interpolation artifacts on the prostate central and bottom parts, when reverse TPS is used (Fig. 7(a)) and a smooth deformation when Shepard's interpolation is applied (Fig. 7(b)).

The algorithms have been implemented in MATLAB with $1.66 \mathrm{GHz}$, Core2Duo processor with 2GB memory. Table II shows that TPS/Shepard's interpolation takes a maximum of 94.58 secs. The NMI computation takes 0.5 secs on an average with the current hardware configuration.

\section{CONCLUSIONS AND Future WORKS}

A novel approach to generate an optimal set of correspondence points automatically for registration, based on the segmented prostate contours has been presented. The method is 
TABLE II

COMPUTATIONAL PERFORMANCE AND ACCURACY OF THE PROPOSED ALGORITHM.

\begin{tabular}{|c|c|c|c|}
\hline \hline Patient\# & $\begin{array}{c}\text { Correspondence } \\
\text { generation }(\text { secs })\end{array}$ & $\begin{array}{c}\text { TPS/Shepard's } \\
\text { method (secs) }\end{array}$ & DSC \\
\hline 1 & 0.2 & 94.58 & 0.98 \\
2 & 0.2 & 83.91 & 0.97 \\
3 & 0.14 & 22.71 & 0.96 \\
4 & 0.16 & 50.15 & 0.97 \\
\hline
\end{tabular}

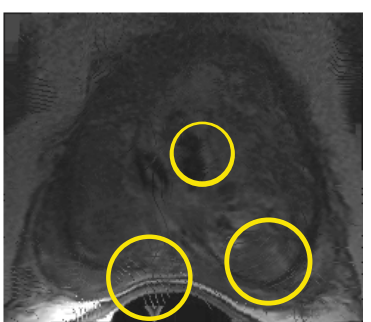

(a) Forward/Reverse TPS

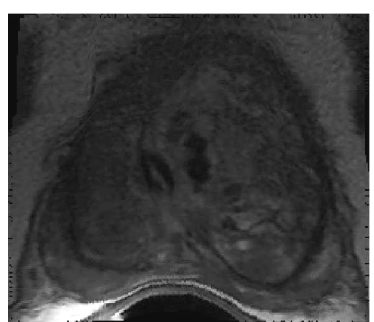

(b) Forward TPS/Shepard
Fig. 7. Comparison of forward/reverse TPS interpolation with forward TPS/Shepard's interpolation, (a) interpolation artifacts visible in center and lower part of the image (encircled), (b) smooth deformation with Shepard's interpolation.

based on triangular approximations of closed boundary regions that may be generalized for regular-shaped anatomical structures. In comparison with other geometric contour sampling methods, our method showed better correspondences being generated resulting in smooth deformations and significantly high region overlaps after TPS registration. The approach of using NMI in determining the optimal set of correspondences has been consistent for all patients and has been proved both qualitatively and quantitatively. TPS interpolation along with Shepard's interpolation produced smoother deformations and could avoid interpolation artifacts resulting from backward TPS transformation.

As future work, we would like to validate our method of correspondence point generation with more patient data in order to claim on the robustness of the algorithm and extend our method to 3D multimodal registration of TRUS and MR volumes. Our method of generating optimal correspondences may be of practical significance during interventional US prostate biopsies if the manual selection of the corresponding US/MR slices is automated and the forward TPS/Shepard's interpolation and the computation of NMI at each resolution of correspondences can be parallelized with GPU implementation.

\section{ACKNOWLEDGMENTS}

This research is a part of the PROSCAN project of the VICOROB laboratory of University of Girona, Catalunya, Spain. The authors would like to thank VALTEC 08-1-0039 of Generalitat de Catalunya, Spain and Conseil Regional de Bourgogne, France for funding this research.

\section{REFERENCES}

[1] C. R. Porter, C. O'Donnell, E. D. Crawford, E. J. Gamito, J. Kim, and A. Tewari, "Predicting the outcome of the random prostate biopsy,"
Department of Urology, State University of New York, Stony Brook,NY., Tech. Rep., http://www.cancernews.com/data/Article/230.asp, accessed [30 May 2010]

[2] I. Kaplan, N. E. Oldenburg, P. Meskell, M. Blake, P. Church, and E. J. Holupka, "Real time MRI-ultrasound image guided stereotactic prostate biopsy," Magnetic Resonance Imaging, vol. 20, pp. 295-299, 2002.

[3] S. Xu, J. Kruecker, B. Turkbey, N. Glossop, A. K. Singh, P. Choyke, P. Pinto, and B. J. Wood, "Real-time MRI-TRUS fusion for guidance of targeted prostate biopsies," Computer Aided Surgery, vol. 13, no. 5, pp. 255-264, 2008.

[4] A. K. Singh, J. Kruecker, S. Xu, N. Glossop, P. Guion, K. Ullman, P. L. Choyke, and B. J. Wood, "Initial clinical experience with real-time transrectal ultrasonography-magnetic resonance imaging fusion-guided prostate biopsy," British Journal of Urology International, vol. 101, no. 7, pp. 841-845, April 2008.

[5] R. Narayanan, J. Kurhanewicz, K. Shinohara, E. D. Crawford, A. Simoneau, and J. S. Suri, "MRI-ultrasound registration for targeted prostate biopsy," IEEE Intl. Symposium on Biomedical Imaging: From Nano to Macro, pp. 991-994, 2009.

[6] J. Lu, R. Srikanchana, M. McClain, Y. Wang, J. Xuan, I. A. Sesterhenn, M. T. Freedman, and S. K. Mun, "A statistical volumetric model for characterization and visualization of prostate cancer," Proc. of SPIE, vol. 3976, pp. 142-153, February 2000.

[7] B. Fei, C. Kemper, and D. L. Wilson, "A comparative study of warping and rigid body registration for the prostate and pelvic MR volumes," Computerized Medical Imaging and Graphics, vol. 27, pp. 267-281, 2003.

[8] M. A. Padilla-Castañeda and F. Arámbula-Cosió, "Resection simulations with local tissue deformations for computer assisted surgery of the prostate," Lecture Notes in Artificial Intelligence, vol. 2313, pp. 450459, 2002.

[9] P. Yan, S. Xu, B. Turkbey, and J. Kruecker, "Optimal search guided by partial active shape model for prostate segmentation in trus images," Proceedings of SPIE Medical Imaging, vol. 7261, pp. 72611G.1$72611 \mathrm{G} .11,2009$.

[10] D. Shepard, "A two-dimensional interpolation function for irregularlyspaced data," Proceedings of the 1968 23rd ACM national conference, pp. 517-524, 1968.

[11] S. Ghose, A. Oliver, R. Martí, X. Lladó, J. Freixenet, J. Vilanova, and F. Meriaudeau, "Texture guided Active Appearance Model propagation for prostate segmentation," Accepted in Prostate Cancer Imaging, Computer Aided Diagnosis, Prognosis and Intervention, MICCAI workshop, 2010.

[12] A. Collignon, F. Maes, D. Delaere, D. Vandermeulen, P. Suetens, and G. Marchal, "Automated multimodality image registration using information theory," Intl. Conf. on Information Processing in Medical Imaging, pp. 287-298, 1995.

[13] F. Bookstein, Morphometric Tools for Landmark Data: Geometry and Biology. Cambridge, UK: Cambridge University Press, 1991.

[14] K. Rohr, H. S. Stiehl, R. Sprengel, T. M. Buzug, J. Weese, and M. H. Kuhn, "Landmark-based elastic registration using approximating thinplate splines," IEEE Transactions on Pattern Analysis and Machine Intelligence, vol. 20, no. 6, June 2001. 